\title{
56 MHZ Cavity Prototype Measurements
}

\author{
H. Hahn, E. Choi
}

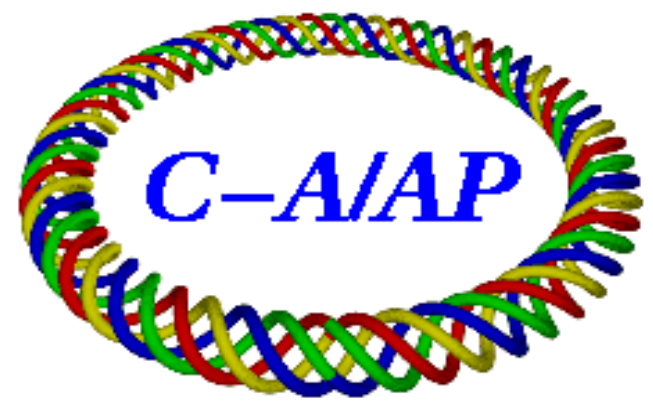

\section{Collider-Accelerator Department Brookhaven National Laboratory Upton, NY 11973}

Notice: This document has been authorized by employees of Brookhaven Science Associates, LLC under Contract No. DE-AC02-98CH10886 with the U.S. Department of Energy. The United States Government retains a nonexclusive, paid-up, irrevocable, world-wide license to publish or reproduce the published form of this document, or allow others to do so, for United States Government purposes. 


\section{MHZ Cavity Prototype measurements}

Harald Hahn and Eunmi Choi

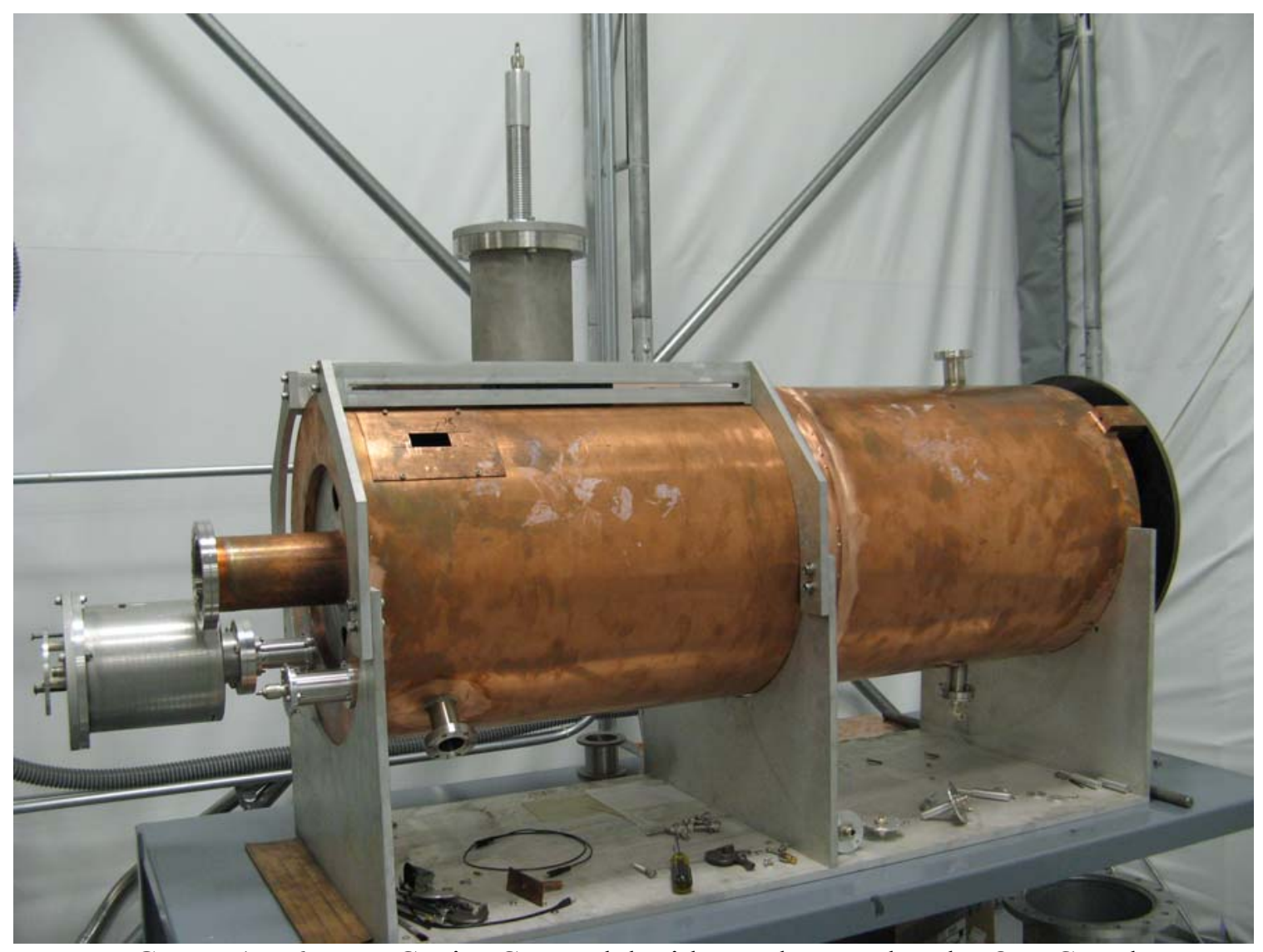

FIGURE 1: $56 \mathrm{MHz}$ Cavity Cu Model with Fundamental and HOM Coupler

\section{MEASUREMENTS PERFORMED}

The following series of measurements were performed on the $\mathrm{Cu}$ model in order to determine the physics requirements and to demonstrate feasibility.

\section{Slow tuner frequency change}

\section{Fundamental Damper}

External Q as function of position

Location of position for minimum frequency change

\section{HOM Damper}

External Q at $56 \mathrm{MHz}$ with single damper

$Q$ values at critical harmonics ( ) with single damper 


\section{Pick-up probe}

Capacitive at acceleration gap

Magnetic at shorted end (postponed for SC measurement)

\section{Self inductance of fundamental damper loop}

\section{INTERPRETATION of MEASUREMENTS}

All measurements involved the use of a network analyzer. The well known difference between accelerator parameters and circuit parameters, provided by the network analyzer, can cause confusion. At first the frequently used equivalent circuit parameters with their definition is given.

\section{Equivalent Circuit Analysis}

SuperFish + Microwave Study provide

- stored energy $U_{C}$ and the power lost in the cavity $P_{C}$ at accelerating field (index $\mathrm{C}$ as in Padamsee and index CD for circuit definition)

$$
V_{C}=V_{C D} \sqrt{2}
$$

- shunt impedance (index A for accelerator definition and SH shunt impedance in circuit definition)

$$
\begin{aligned}
& R_{A}=V_{C}^{2} / P_{C} \text { and } \\
& R_{S H}=V_{C D}^{2} / P_{C}=R_{A} / 2
\end{aligned}
$$

- intrinsic quality factor $Q_{0}=\frac{\omega_{0} U_{C}}{P_{C}}$ yielding the cavity inductance

$$
L=\frac{1}{2 \omega_{0}}\left(\frac{R_{A}}{Q_{0}}\right)
$$

In order to ovoid errors and to simplify the interpretation of measurements, a list of typical measurements is given here.

\section{The port coupler strength}

The coupler strength characterized in terms of $\beta$ or transformer ratio $n: 1$ is obtained from an 21 measurement which yields the impedance

$$
R_{\text {Port }}=R_{0} \frac{1-S_{21}}{1+S_{21}} \text { with } R_{0}=50 \Omega
$$

from which follows

$$
\beta=\frac{R_{\text {Port }}}{R_{S H}} \text { and } n=\sqrt{\frac{R_{S H}}{R_{\text {Port }}}}
$$

Note that once measured at room temperature $n$ remains constant during cool-down. The accuracy from the S21 measurements is reduced for a weak coupler such as a pickup probe and must by replaced by the following measurement of the Q-external . 


\section{Measurement of Q-external}

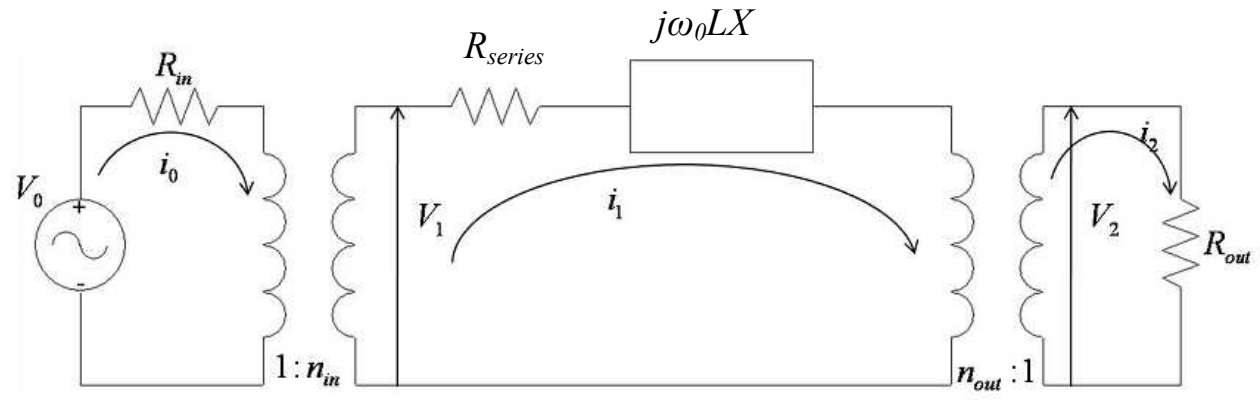

$$
S_{21}=\frac{2 V_{2}}{V_{0}}=\frac{2 n_{\text {in }} n_{\text {out }} R_{\text {out }}}{R_{\text {Series }}+n_{i}^{2} R_{\text {in }}+n_{o}^{2} R_{\text {out }}+j \omega_{0} L \Delta}
$$

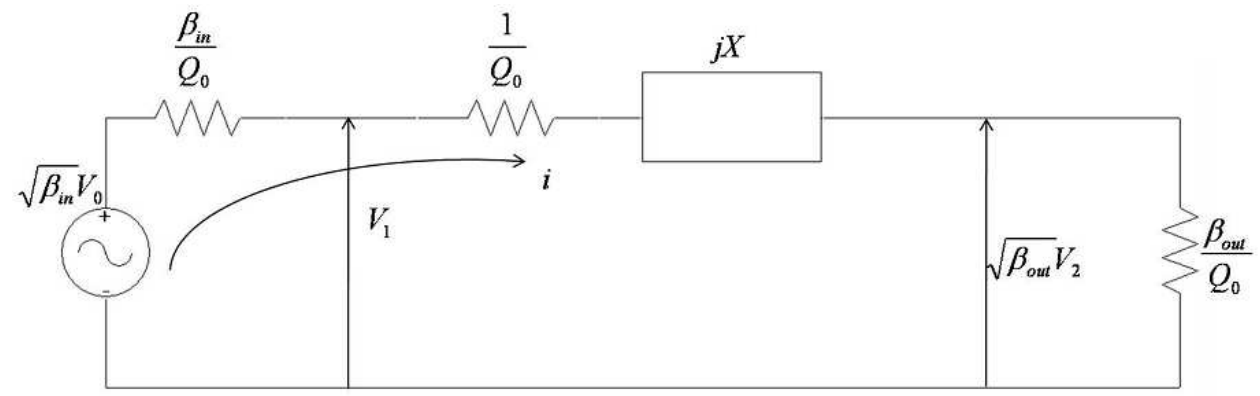

$$
S_{21}=\frac{2 \sqrt{\beta_{\text {in }} \beta_{\text {out }}}}{1+\beta_{\text {in }}+\beta_{\text {out }}+Q_{0} \Delta}=\frac{2 Q_{L}}{\sqrt{Q_{\text {in }} Q_{\text {out }}}\left(1+j Q_{L} \Delta\right)}
$$

Convenient method to find Q-external $\left(Q_{\text {ext }}=Q_{\text {out }}\right.$ ) involves critically coupled $Q_{\text {in }}=Q_{0}$ Yielding at resonance, with $\Delta=0$ and $Q_{L}=Q_{0} / 2$,

$$
Q_{\text {ext }}=Q_{0} / S_{21}^{2}
$$

\section{The dissipated power to the (external) load}

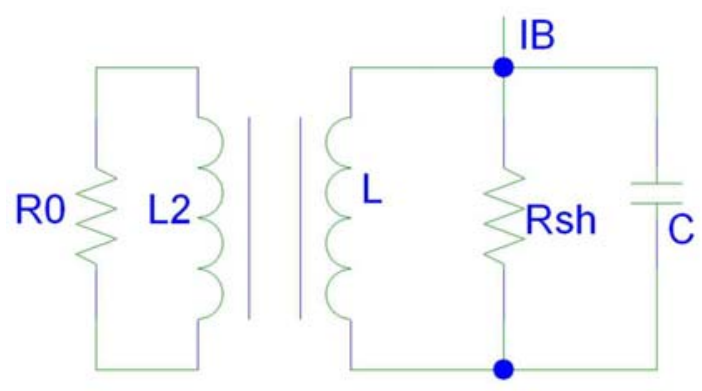

$\mathrm{M}$

Ignoring the bunch length, the time averaged beam current $I_{B}$ produces a cavity voltage

$$
V_{C D}=Z_{C D} \frac{2 I_{B}}{\sqrt{2}}
$$


The generic cavity impedance with $L, C, R_{T}$ in circuit definition is

$$
\begin{aligned}
& Z_{C D}=\frac{\omega_{0} L Q_{L}}{1+j Q_{L} \Delta}=\frac{R_{L}}{1+j Q_{L} \Delta} \text { with } \Delta=\frac{\omega}{\omega_{0}}-\frac{\omega_{0}}{\omega} \\
& \frac{1}{R_{L}}=\frac{1}{R_{S H}}+\frac{1}{R_{X}}
\end{aligned}
$$

The total power into the cavity

$$
P_{T}=2 \frac{Z_{C D}^{*} Z_{C D}}{R_{L}} I_{B}^{2}=2 \frac{R_{S H}}{Q_{0}} \frac{Q_{L}}{1+\left(Q_{L} \Delta\right)^{2}} I_{B}^{2}
$$

The external power

$$
P_{X}=\frac{Q_{L}}{Q_{X}} P_{L}=2 \frac{R_{S H}}{Q_{X}} \frac{Q_{L}^{2}}{1+\left(Q_{L} \Delta\right)^{2}} I_{B}^{2}
$$

\section{Cavity Mutual inductance $M$ and Loop Self Inductance $L_{2}$}

Determining the self inductance of the loops and mutual inductance of the FPC and the HOM coupler presented a specially difficult task not easily solved by theory.

$M$ and $L_{2}$ were obtained from a S11 measurement and its application to the following circuit

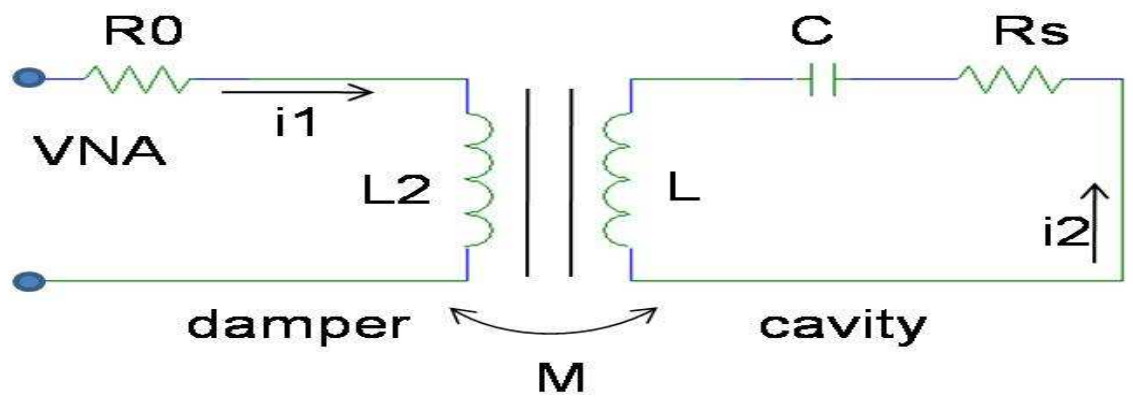

The Vector Network Analyzer provides input impedance

$$
Z=R_{0} \frac{1-S_{11}}{1+S_{11}}
$$

which is follows from the circuit as

$$
Z=j \omega L_{2}+\frac{(\omega M)^{2}}{R_{S}+j \omega_{0} L \Delta} \text { with } \Delta=\left(\frac{\omega}{\omega_{0}}-\frac{\omega_{0}}{\omega}\right)
$$

In terms of the series resistor $R_{S}=\omega_{0} L / Q_{0}$ and $L=\frac{1}{2 \omega_{0}}\left(\frac{R_{A}}{Q_{0}}\right)$ already as defined above

One finds the expressions for the real part and imaginary part as follows,

$$
\begin{aligned}
& \operatorname{Re} Z=\frac{(\omega M)^{2}}{R_{S}^{2}+\left(\omega_{0} L \Delta\right)^{2}} \\
& \operatorname{Im} Z=\omega L_{2}-\frac{\omega_{0} L \Delta(\omega M)^{2}}{R_{S}^{2}+\left(\omega_{0} L \Delta\right)^{2}}
\end{aligned}
$$


At resonance, $\operatorname{Re} Z$ will give the mutual inductance $M$, and

$\operatorname{Im} Z$ will give the self inductance $L_{2}$ separately.

The results for the fundamental Damper are

$M \approx 11 \mathrm{nH}$ and $L_{2}=261 \mathrm{nH}$, the latter being a surprisingly large value.

\section{References}

1. H. Padamsee, J. Knobloch, and T. Hays, RF Superconductivity for Accelerators, (John Wiley \& Sons, 1998)

2. E. M. Choi and H. Hahn, Summary of the fundamental mode damper experiments of the $56 \mathrm{MHz} S R F$ cavity, BNL Report C-AD/AP/314, July 2008

3. E. M. Choi and H. Hahn, Capacitive fundamental power coupler and pickup for the $56 \mathrm{MHz}$ SRF cavity, BNL report C-AD/AP/315, July 2008

4. E. M. Choi and H. Hahn, Higher Order Mode Damper Study of the $56 \mathrm{MHz} S R F$ cavity, BNL Report C-AD/AP/319, August 2008 